\title{
Clear Cell Sarcoma of Right Knee with Bone Marrow Metastasis: A Case Report and review of Literature
}

\author{
Divya Shelly*, Shashank Mishra, Divya Gupta and Reena Bharadwaj \\ Department of Pathology Armed Forces Medical College, Pune.
}

\begin{abstract}
Clear cell sarcoma (CCS) of the soft tissue is an extremely rare malignant tumor which was first described by Dr Franz M Enzinger in 1965. Soft tissue sarcomas comprise only $1 \%$ of malignant tumors and clear cell sarcoma is the rarest among these. It is closely associated with tendons, aponeurosis and fascial structures of extremities. Histomorphologically and immunohistochemically it resembles amelanotic melanoma and was earlier called as malignant melanoma of soft parts. But it is a unique soft tissue tumour with uninvolved overlying skin and characteristic chromosomal translocation. The characteristic histopathological features along with immunohistochemical positivity for S-100p, Melan-A and HMB-45 help in differentiation of this tumour from other lesions like fibrosarcoma and MPNST. The common site of metastasis includes lymph nodes, lungs, skin, liver, heart and brain. We present a rare case of an 18-year-old boy with clear cell sarcoma of right knee, which had lymph node metastasis at presentation and found to have bone marrow metastasis during the course of the disease.
\end{abstract}

Keywords: Clear Cell Sarcoma, Soft Tissue, Amelanotic Melanoma, Bone Marrow Metastasis

\section{Introduction}

Clear Cell Sarcoma (CCS) of soft tissue, previously referred to as melanoma of soft parts is a rare malignant tumor arising from soft tissues around tendons and aponeurosis of distal extremities. Rarely CCS has also been reported in bone, gastro-intestinal tract and head and neck. It was first described by Frank H Enzinger in 1965 when he published a case series of 21 cases which described the clinical and histomorphological characteristics of this distinct entity. ${ }^{[1]}$ This soft tissue sarcoma is derived from neural crest cells and accounts for less than $1 \%$ of soft tissue tumors. [2] Due to its morphological, immunohistochemical and ultrastructural similarity to malignant melanoma, it is often difficult to distinguish it from malignant melanoma. [3] Clinically, CCS presents as a slowly progressive and painless mass in young adults with a predilection for the extremities. The tumour increases in size and metastatic dissemination to lymph nodes and lungs is seen. We are describing here a case of clear cell sarcoma of right knee, which had lymph node metastasis at presentation and found to have bone marrow metastasis during the course of the disease.

\section{Case Report(S)}

An 18-year-old male presented to our hospital with a painless soft tissue swelling around anterior aspect of the right knee of one-year duration. Physical examination revealed a subcutaneous swelling which was firm, nontender and measured $3 \times 1.2 \times 1 \mathrm{cms}$ in size. MRI showed the presence of a well-defined enhancing lesion measuring 3 X 1.3 X $1.2 \mathrm{cms}$ which showed signal enhancement on
T1 weighted images. During preoperative evaluation, right inguinal group of lymph nodes was found to be enlarged and so fine needle aspiration (FNAC) was done from this node to rule out metastasis. FNAC smears revealed metastatic deposits of tumour composed of neoplastic oval to spindle shaped cells arranged in small clusters and scattered singly, having prominent red inclusion-like nucleoli. The presence of these oval to spindle shaped cells with large nucleoli raised the suspicion of melanoma. (Fig 1) However, in view of soft tissue lesion around the knee, the possibility of metastasis from clear cell sarcoma was suggested.

Subsequently, wide local excision specimen of the soft tissue tumour was received along with inguinal, femoral and iliac group of lymph node dissection. Gross examination revealed a skin covered tissue bit measuring $7 \times 2.8 \times 1.2 \mathrm{cms}$ which showed the presence of a well-demarcated tan-grey, firm mass in the dermis and subcutaneous tissue measuring $3 \times 1.2 \times 1.1 \mathrm{cms}$. Hematoxylin and eosin stained sections from the lesion showed the presence of a tumour located in the dermis and subcutaneous soft tissue. The tumour was arranged in nests, lobules and alveolar pattern separated by thin fibrous septae. Overlying epidermis was normal and no junctional activity was noted. (Fig 2A) Tumour cells were round to polygonal with moderate clear to light eosinophilic cytoplasm, mildly pleomorphic vesicular nuclei with prominent inclusion-like nucleoli. (Fig 2B) Scattered multinucleated giant cells and areas of necrosis were seen. Mitosis was frequent. Eight out of eighteen dissected lymph nodes showed the presence of metastatic 
tumour deposits. On immunohistochemistry, tumour cells were diffusely positive for S-100p, Melan-A, and HMB45, while were negative for CD34, CD31, desmin, SMA, CD68, CD163 and LCA (Fig 3A and B). Tumour retained INI-1 expression. Based on the histomorphological features and immunohistochemical profile diagnosis of clear cell sarcoma was rendered. Post-operatively, the patient underwent $50 \mathrm{~Gy} / 25 \#$ of radiotherapy. Post-radiotherapy, the patient developed bicytopenia in the form of anaemia and leukopenia. Bone marrow biopsy revealed nests of epithelioid cells and areas of necrosis, thus confirming deposits of CCS (Fig 4A and B). The patient was started on cisplatin and doxorubicin-based chemotherapy; however, he expired within two days of initiation of therapy.

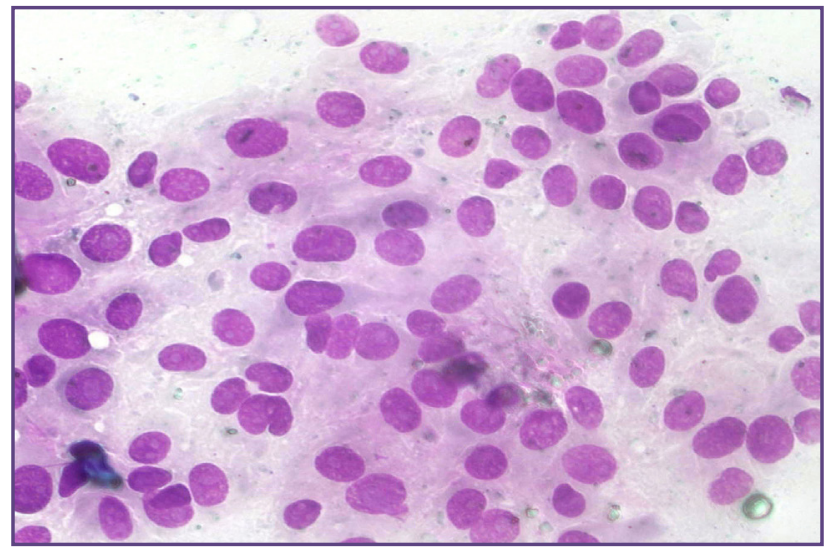

Fig. 1: FNAC from right inguinal lymph node showing oval to fusiform shaped tumour cells in clusters. These cells have moderate cytoplasm, pleomorphic nuclei with inclusion like nucleoli (MGG, 400X).

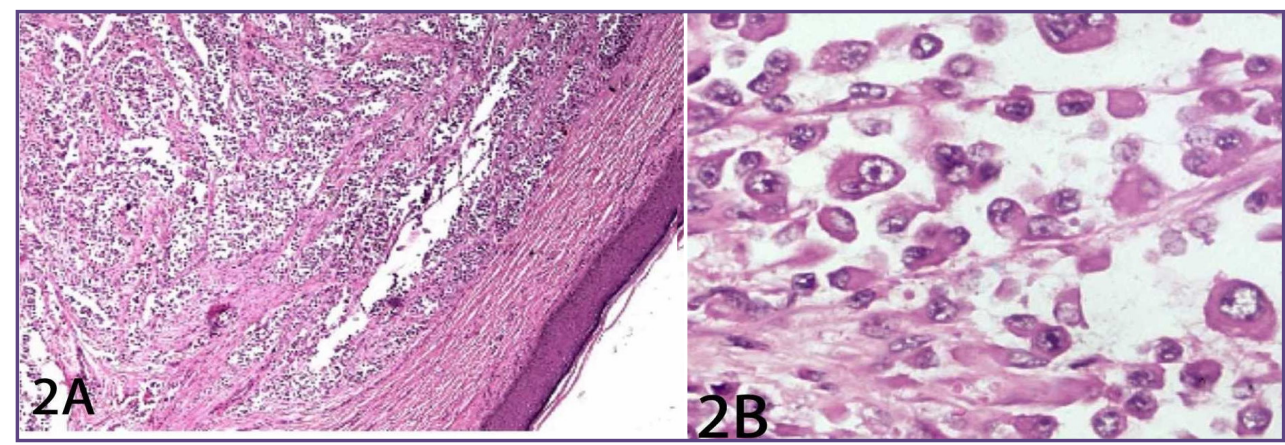

Fig. 2: (A) Section showing a tumour in the dermis and subcutaneous tissue with normal overlying epidermis. Tumour cells are arranged in nests and alveolar pattern divided by thin fibrous septae (H\&E,20X) 2 (B) Section showing pleomorphic oval to fusiform tumour cells having moderate cytoplasm, pleomorphic nuclei with vesicular chromatin and prominent red nucleoli (H\&E,400X).
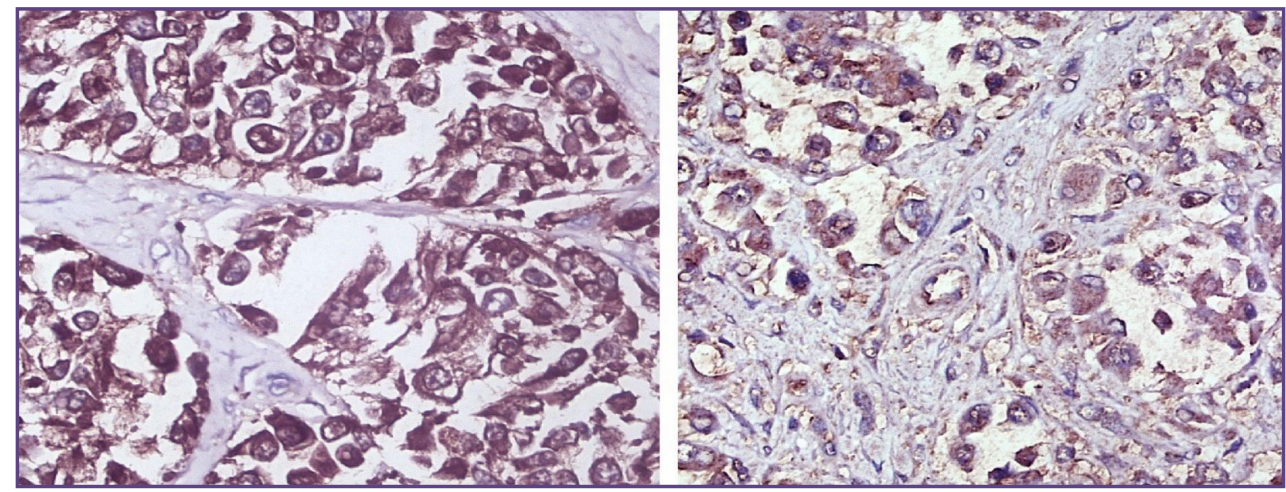

Fig. 3: (A) The tumour cells show diffuse nuclear and cytoplasmic positivity for S-100p (H\&E, 400X) and 3 (B) cytoplasmic positivity for HMB 45 (H\&E, 400X). 


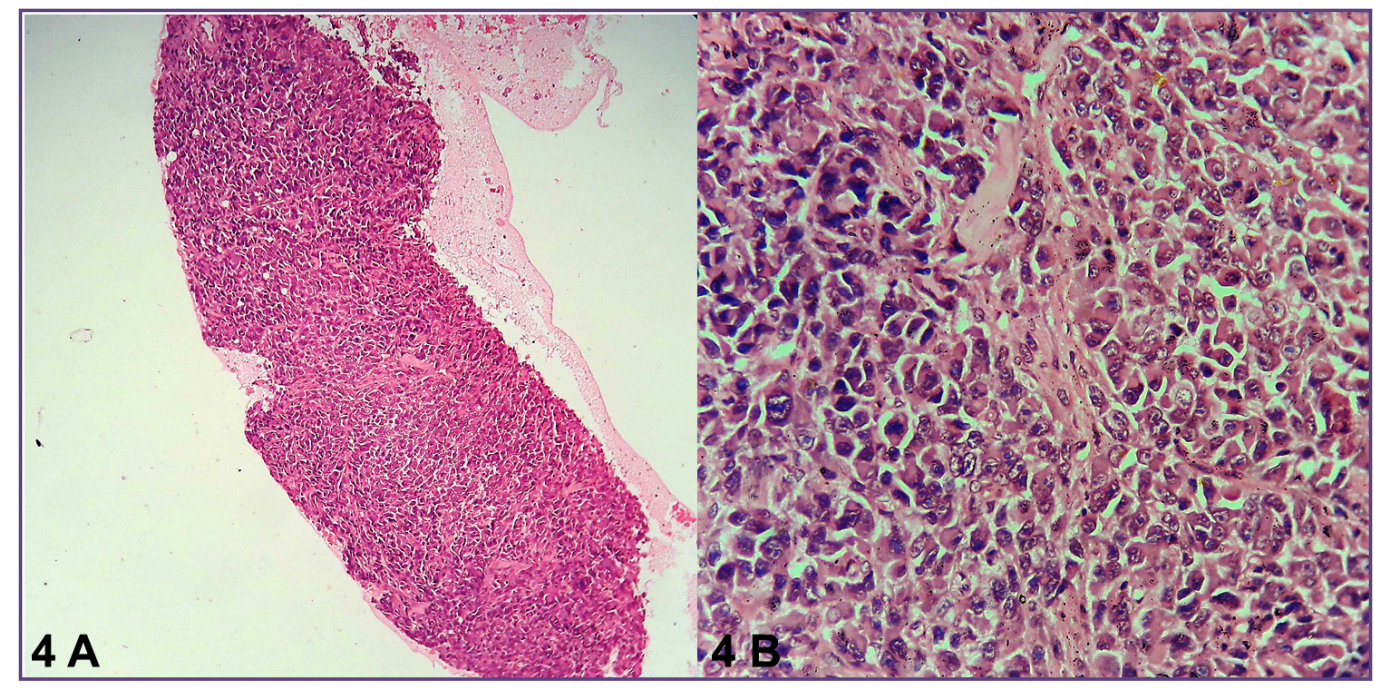

Fig. 4: (A) Section showing near total replacement of bone marrow by sheets of tumour cells. (H\&E, 20X) 4 (B) Section showing pleomorphic epithelioid tumour cells having moderate eosinophilic cytoplasm, pleomorphic nuclei with vesicular chromatin and prominent nucleoli (H\&E, 400X).

\section{Discussion}

Clear cell sarcoma (CCS) of soft tissue is currently a distinct entity classified by the World Health Organization as soft tissue and bone tumours. ${ }^{[4]}$ The tumour has an age range of 2-82 years with a median age of 27 years. ${ }^{(2)}$ The principal sites of the tumour are the extremities, especially the region of the foot and ankle. Cases of clear cell sarcoma have also been reported in around knee, thigh, hand, forearm, elbow, shoulder, head and trunk in decreasing order of frequency. ${ }^{[6]}$ Grossly, CCS appears as a localised, tan-grey coloured circumscribed mass in deep soft tissue. Tumour is usually small in size at presentation with more than half of all cases measuring less than $4 \mathrm{~cm} .{ }^{(1)}$ Our patient presented with soft tissue mass around the knee measuring $3 \mathrm{~cm}$ in size. On MRI, CCS is usually homogenous strongly enhancing soft tissue mass which is generally well defined and benign looking. Thus, imaging may be non-specific and definite diagnosis can only be made on histopathology. ${ }^{[7]}$

Microscopically, CCS is composed of infiltrative epithelioid to spindle-shaped cells arranged as compact nests, fascicles and in the alveolar pattern. ${ }^{[2]}$ These nests are separated by fibrous septae which are often continuous with collagen of tendons and aponeurosis. Tumour cells have ill-defined cell borders with a moderate amount of clear to pale amphophilic cytoplasm. Clear appearance of cytoplasm is due to accumulation of intracellular glycogen which can be demonstrated with periodic acid-Schiff (PAS) and PAS with diastase (PAS-D) stains. Tumour cells show minimal pleomorphism and low mitotic activity which is in concordance with the slow-growing behavior of the tumor. Scattered multinucleated tumor giant cells are commonly present and areas of focal necrosis may be noted. Nuclei are round with finely dispersed chromatin and large prominent nucleoli. ${ }^{[1]}$ Biphasic differentiation should be absent. In our case, most of the tumor cells were epithelioid with few areas of spindled morphology. The cytoplasm of the tumor cells was mainly palely eosinophilic and only a minority of tumor cells showed true clearing cytoplasm, which is a common cytological feature of this kind of tumor.

On Immunohistochemistry, CCS cells express antigens associated with melanin synthesis including a diffuse cytoplasmic immunoreactivity with HMB-45, nuclear and cytoplasmic immunoreactivity for S-100 protein and reactivity with microphthalmia transcription factor (MITF) and Melan-A (figure 3). These markers help in differentiating this tumour from epithelial malignancies and synovial sarcoma. Diagnosis of CCS can be confirmed by the presence of a unique and recurrent $t(12 ; 22)$ (q13;q12) involving the fusion of the EWS gene on 22q12 with the ATF1 gene on 12q13, which is identified in $70 \%$ $90 \%$ of clear cell sarcomas. ${ }^{[8]}$ Testing for this translocation can be done by cytogenetics, reverse-transcriptase polymerase chain reaction (RT-PCR) and fluorescence in situ hybridization (FISH). ${ }^{[9]}$ This translocation is not observed in cutaneous melanomas, fibrosarcomas and malignant peripheral nerve sheath tumor (MPNST) ${ }^{[4]}$ Molecular studies could not be performed in this case due to practical constraints. 
Apart from malignant melanoma another differential diagnosis that should be kept in mind while evaluating a tumor located in proximity to tendons and aponeurosis includes paraganglioma-like dermal melanocytic tumour, clear cell myelomonocytic tumour, epithelioid malignant peripheral nerve sheath tumour and monophasic synovial sarcoma. Paraganglioma-like dermal melanocytic tumour presents as a dermal nodule of extremities in females. It has a paraganglioma-like an arrangement of clear to amphophilic oval cells with the low nuclear grade. Unlike CCS, paraganglioma-like dermal melanocytic tumour does not show EWS gene locus rearrangement. ${ }^{[10]}$ Clear cell myelomelanocytic tumor is an intra-abdominal neoplasm of young adults and commonly arises from falciform ligament and ligamentam teres of liver. Clear cell myomelanocytic tumor belongs to the perivascular epithelioid cell family of tumors (PEComas). ${ }^{[11]}$ Histologically, they appear as nests and fascicles of uniform spindle cells with indistinct nucleoli which lack multinucleation. Malignant peripheral nerve sheath tumor usually arises from a large peripheral nerve. Tumour cells are spindle to epithelioid in shape with hyperchromatic nuclei against a myxoid stroma. Unlike CCS, mitotic activity is brisk and cells are negative for melanocytic markers. No recurring cytogenetic abnormality has been identified in malignant peripheral nerve sheath tumors. Monophasic synovial sarcoma can be excluded by characteristic immunohistochemical positivity for bcl-2 and CD99 and negativity for melanocytic markers like HMB45 and Melan A. Synovial sarcoma has a characteristic and diagnostic chromosomal translocation $\mathrm{t}(\mathrm{X} ; 18)(\mathrm{p} 11.2 ; \mathrm{q} 11.2)$ or by SYTSSX1 or SYT/SSX2 gene fusion detected by RT-PCR and FISH. ${ }^{[12]}$

Lymph nodes are the most common site of metastasis and have been reported in a high number of cases. The presence of lymph node metastasis is associated with a poor survival In our case, the patient had inguinal node metastasis at presentation and diagnosis was suspected on FNAC of the inguinal node. Lungs, skin, liver, heart and brain are other sites of distant metastasis. However, our patient also developed bone marrow metastasis, which is the rarely described in the literature.

Wide surgical resection is the treatment of choice for clear cell sarcoma. Adjuvant chemotherapy is not necessary if complete excision is achieved. In cases having vascular or neural impairment amputation is justified. Postoperative radiation therapy is given when the tumour is close to surgical resection margins. Cisplatin and doxorubicinbased chemotherapy are needed in patients with metastatic disease and with a tumour size more than $5 \mathrm{cms} .{ }^{[13]} \mathrm{CCS}$ patients have a variable and unpredictable clinical course. A tumour size of $>5 \mathrm{~cm}$ is an independent prognostic factor and is associated with a poor survival and high incidence of distant metastasis. ${ }^{[2]}$ Preoperative duration of symptoms, mitotic index or vascular invasion may not predict survival in these patients. The 5- and 10-year overall survival rates are $59 \%$ and $41 \%$, respectively. ${ }^{[7]}$

\section{Conclusion}

To conclude Clear cell sarcoma of soft tissue is a rare malignant soft tissue tumour which must be kept in the differential diagnosis of soft tissue tumors involving extremities in young patients. Although the tumour may appear benign on imaging studies, $50 \%$ of patients may present with nodal metastasis. Other sites of distant metastasis include lungs, skin, liver, heart, brain and rarely bone marrow.

\section{Reference}

1. Enzinger FM. Clear-cell sarcoma of tendons and aponeuroses. An analysis of 21 cases. Cancer. 1965;18(9):1163-74.

2. Mavrogenis AF. Clinicopathological features, diagnosis and treatment of clear cell sarcoma/mela- noma of soft parts. Hippokratia. 2013;17(4):298-302.

3. Hantschke M, Mentzel T, Rütten A, Palmedo G, Calonje E, Lazar AJ, et al. Cutaneous clear cell sarcoma: a clinicopathologic, immunohistochemical, and molecular analysis of 12 cases emphasizing its distinction from dermal melanoma. Am J Surg Pathol. 2010;34(2):216-22.

4. Hisaoka M, Ishida T, Kuo T-T, Matsuyama A, Imamura T, Nishida K, et al. Clear cell sarcoma of soft tissue: a clinicopathologic, immunohistochemical, and molecular analysis of 33 cases. Am J Surg Pathol. 2008;32(3):452-60.

5. Doyle LA. Sarcoma classification: an update based on the 2013 World Health Organization Classification of Tumors of Soft Tissue and Bone. Cancer. 2014;120(12):1763-74.

6. Goldblum J, Folpe A, Weiss S. Enzinger And Weiss's Soft Tissue Tumors. Sixth. Elsevier Saunders; 2013. p886-897

7. Hocar O, Le Cesne A, Berissi S, Terrier P, Bonvalot S, Vanel D, et al. Clear Cell Sarcoma (Malignant Melanoma) of Soft Parts: A Clinicopathologic Study of 52 Cases. Dermatol Res ;2012:1-8.

8. De Beuckeleer LH, De Schepper AM, Vandevenne JE, Bloem JL, Davies AM, Oudkerk M, et al. MR imaging of clear cell sarcoma (malignant melanoma of the soft parts): a multicenter correlative MRI-pathology study of 21 cases and literature review. Skeletal Radiol. 2000;29(4):187-95.

9. Patel RM, Downs-Kelly E, Weiss SW, Folpe AL, Tubbs RR, Tuthill RJ, et al. Dual-color, break-apart fluorescence in situ hybridization for EWS gene rearrangement distinguishes clear cell sarcoma of soft tissue from malignant melanoma. Mod Pathol. 2005;18(12):1585-90. 
10. Deyrup AT, Althof P, Zhou M, Morgan M, Solomon AR, Bridge JA, et al. Paraganglioma-like dermal melanocytic tumor: a unique entity distinct from cellular blue nevus, clear cell sarcoma, and cutaneous melanoma. Am J Surg Pathol. 2004;28(12):1579-86.

11. Folpe AL, Goodman ZD, Ishak KG, Paulino AF, Taboada EM, Meehan SA, et al. Clear cell myomelanocytic tumor of the falciform ligament/ligamentum teres: a novel member of the perivascular epithelioid clear cell family of tumors with a predilection for children and young adults. Am J Surg Pathol. 2000;24(9):1239-46.

12. Dim DC, Cooley LD, Miranda RN. Clear Cell Sarcoma of Tendons and Aponeuroses A Review. Arch Pathol Lab Med-Vol. 2007;131:152-56.

13. Mankin HJ, Hornicek FJ. Diagnosis, classification, and management of soft tissue sarcomas. Cancer Control. 2016;12(1):5-21.

\section{*Corresponding author:}

Surg CDr Divya Shelly, Assistant Professor, Department of Pathology, Armed Forces Medical College, Sholapur Road, Pune-411040 INDIA

Phone: +919930676695

Email: dshelly13@gmail.com

Date of Submission : 12.10.2016

Date of Acceptance : 21.11.2016

Financial or other Competing Interests: None.

Date of Publication : 19.02.2017 